\title{
Renormalization group study of a two-valley system with spin-splitting
}

\author{
Alexander Punnoose \\ Physics Department, City College of the City University of New York, New York, NY 10031, USA*
}

\begin{abstract}
Renormalization group equations in a two-valley system with valley-splitting and intervalley scattering are derived in the presence of spin-splitting induced by a parallel magnetic field. The relevant amplitudes in different regimes set by the relative strengths of the spin and valley splittings and the intervalley scattering rate are identified. The range of applicability of the standard formula for the magnetoconductance is discussed.
\end{abstract}

PACS numbers: 72.10.-d, 71.30.+h, 71.10.Ay

\section{INTRODUCTION}

In two dimensions, an in-plane magnetic field, $B_{\|}$, conveniently couples only to the spin-degrees of freedom leading to spin-splitting of the electron bands. Electron-electron $(e-e)$ interactions between the different spin-bands gives rise to a finite magnetoconductance, $\sigma\left(B_{\|}, T\right)$, and hence measurement of $\sigma\left(B_{\|}, T\right)$ provides a simple and accurate way of determining the effective spin-related interaction strength ${ }^{1,2}$. In a disordered two-dimensional electron gas (2DEG), the transport properties at low temperatures, $k_{B} T \lesssim \hbar / \tau$, are governed by singular diffusive particle-hole propagators ${ }^{3}$, $\mathcal{D}(q, \omega)=1 /\left(D_{0} q^{2}+\omega\right)$. (Here $D_{0}$ is the diffusion constant proportional to the elastic scattering rate $1 / \tau$.) The in-plane magnetic field suppresses the singularity in the spin-triplet channels $\left(S_{z}= \pm 1\right)$ with opposite spin projections by introducing a gap proportional to the Zeeman energy scale $\Delta_{z}=g \mu_{B} B_{\|}$. This suppression gives rise to a negative magnetconductance, $\Delta \sigma\left(B_{\|}, T\right)=\sigma\left(B_{\|}, T\right)-$ $\sigma(0, T)<0$ in the weak-field limit ${ }^{4,5}, \Delta_{z} \lesssim k_{B} T$. In the high-field limit, $\Delta_{z} \gg k_{B} T$, the spin-bands are well split and the transport is governed entirely by the $S_{z}=0$ channels which are insensitive to spin-splitting ${ }^{6-8}$. In a multivalley system, valley splitting, $\Delta_{v}$, and intervalley scattering $\Delta_{\perp} \equiv \hbar / \tau_{\perp}$, introduces additional gaps ${ }^{9,10}$ further suppressing $\sigma\left(B_{\|}, T\right)$. The interplay of spin and valley degrees of freedom in the presence of finite $\Delta_{v}$ and $\Delta_{\perp}$ are studied in this paper.

It is now well understood that the singular nature of $\mathcal{D}(q, \omega)$ leads to a strong enhancement of the $e-e$ scattering amplitudes at low energies ${ }^{11}$. In two dimensions, renormalization group ( $R G$ ) theory applied to a weakly disordered system has been extremely successful at capturing this scale dependence to all orders in the $e$-e scattering amplitudes ${ }^{8,12}$. Strong $e-e$ scattering and energy renormalization effects, where the latter takes into account the renormalization of the Stoner enhancement factor, were incorporated into $\Delta \sigma\left(B_{\|}, T\right)$ in Refs. 13 and 14 . They are generalized here to include the effects of $\Delta_{v}$ and $\Delta_{\perp}$ in a two-valley system. [It is assumed that $\Delta_{\perp}<\Delta_{v}$, which appears to be the case in high mobility silicon inversion layers ${ }^{15,16}$.] The relevant interaction amplitudes are identified and the corresponding scaling equations are determined in low, $\Delta_{z} \lesssim \Delta_{\perp}$, and intermediate-fields,
$\Delta_{\perp} \lesssim \Delta_{z} \lesssim \Delta_{v}$. The high-field limit $\Delta_{z}>\Delta_{v}$, has been studied in great detail in Ref. 17. [Note that for $k_{B} T \lesssim \Delta_{z}, \Delta_{v}$, the results in the high and intermediate field regimes are the same provided the spin and valley indices are interchanged.]

The RG equations for finite $\Delta_{v}$ and $\Delta_{\perp}$ with $\Delta_{z}=$ 0 are detailed in Ref. 18. For finite $\Delta_{z}$, it is convenient to work in the (spin-singlet/triplets) $\otimes$ (valleysinglet/triplets) basis so that the spin and valley degrees of freedom are treated on equal footing in the particlehole channels. The diffusion propagators and the $e-e$ scattering amplitudes in this basis are discussed below.

Diffusion modes: For $\Delta_{z}=0$, it was sufficient to label the modes in terms of the valley states $\mathcal{D}_{\alpha}(q, \omega)$, where $\alpha= \pm$ and $\perp$. (See Ref. 18 for further details.) $\alpha=+$ refers to the valley-singlet channel which is gapless, and $\alpha=-$ and $\perp$ refer to the gapped valley-triplet channels with gaps proportional to $\Delta_{\perp}$ and $\Delta_{v}+\Delta_{\perp}$, respectively. Since $\mathcal{D}_{-}$involves scattering only within the same valley, it is insensitive to the splitting $\Delta_{v}$. It, however, develops a gap $\Delta_{\perp}$ when intervalley scattering mixes the two valleys. All modes are effectively gapless at high temperatures, $k_{B} T \gtrsim \Delta_{v}, \Delta_{\perp}$. [Temperature units $T_{v}, T_{\perp}$ and $T_{z}$ will be used interchangeably in the following to represent the scales $\Delta_{v}, \Delta_{\perp}$ and $\Delta_{z}$, respectively.]

For $\Delta_{z} \neq 0$, the spin-triplet channels $S_{z}= \pm 1$ develop spin-gaps proportional to $\Delta_{z}$. Hence, it is convenient to label the propagators as $\mathcal{D}_{t \alpha}$ and $\mathcal{D}_{s \alpha}^{\sigma}$, where the subscript $t$ corresponds to the spin-triplet channels with $S_{z}= \pm 1$, and $s$ labels the $S_{z}=0$ channels, with the singlet and the triplet $S_{z}=0$ channels labeled by $\sigma= \pm$.

e-e scattering amplitudes: In a single valley system, the $e-e$ scattering amplitudes are uniquely described by the spin texture of the scattering channel. The amplitudes $\Gamma_{s}$ and $\Gamma_{t}$ are used to describe the scattering of particle-hole pairs in the spin-singlet and triplet channels, respectively. They are related to the standard static Fermi-liquid amplitudes $\Gamma_{1}$ and $\Gamma_{2}$ as $\Gamma_{s}=\Gamma_{1}-\Gamma_{2} / 2$ and $\Gamma_{t}=-\Gamma_{2} / 2$. These definitions are easily extended to ${ }^{18}$, $\Gamma_{s \alpha}^{\sigma}=\Gamma_{2 \alpha}-4 \Gamma_{1 \alpha}^{\sigma}$ and $\Gamma_{t \alpha}=\Gamma_{2 \alpha}$, where $\alpha= \pm, \perp$, and $\sigma= \pm$. [For notational convenience, the amplitudes $\Gamma_{s \alpha}^{\sigma}$ are defined with a factor of -4.] Note that the intervalley scattering amplitudes $\Gamma_{1 \perp}^{\sigma}$ are generally negligibly small in a clean system because the Coulomb scattering involving large momentum $Q_{0}$ perpendicular to the 
2D plane is suppressed when the width of the inversion layer is many times larger than the lattice spacing, hence $\Gamma_{s \perp}^{\sigma}=\Gamma_{t \perp}=\Gamma_{2 \perp}$. Together, the total number of amplitudes equal $\Gamma_{s \alpha}^{\sigma}\{4\}+\Gamma_{t \alpha}\{4\}+\Gamma_{s \perp}^{\sigma}\{4\}+\Gamma_{t \perp}\{4\}=\{16\}$; the number in brackets denotes the number of channels.

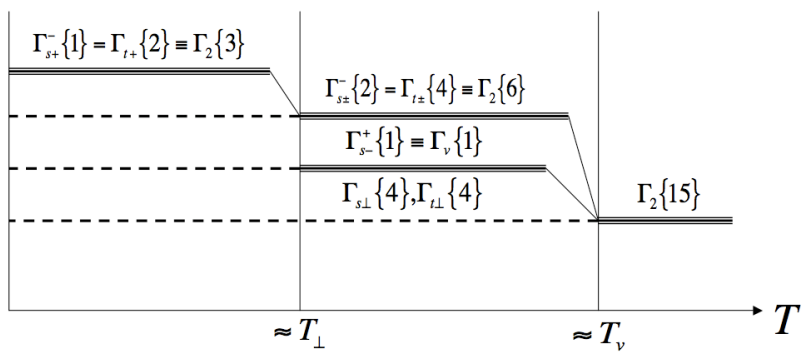

FIG. 1: Schematics showing the classification of the $e-e$ scattering amplitudes as a function of temperature $T$ in the presence of valley splitting $T_{v}$ and intervalley scattering $T_{\perp}$. The relevant amplitudes are marked by solid lines with the degenerate amplitudes grouped together, while dashed lines mark the irrelevant amplitudes. The spin and valley structure of the amplitudes are described in the main text.

In the high temperature limit, $T \gtrsim T_{v}, T_{z}$, the amplitudes $\Gamma_{1 \alpha}^{\sigma}$, except for $\Gamma_{1+}^{+}$, are identically zero. The $\Gamma_{1+}^{+}$amplitude, which involves scattering in the spin and valley singlet channels, (spin-singlet) $\otimes$ (valley-singlet), is special in that it combines with the long-ranged part of the Coulomb interaction to produce a universal amplitude $^{3}$. [Details are given below in section II A.] Hence, all 15 of the 16 amplitudes are equal and evolve as $\Gamma_{2}$. They are shown grouped together when $T \gtrsim T_{v}$ in Fig. 1 .

When $T_{\perp} \lesssim T \lesssim T_{v}$, the $\mathcal{D}_{\perp}$ propagators are gapped, the corrections to $\Gamma_{\perp}$ are therefore non-singular and hence irrelevant. On the other hand, the $\Gamma_{1-}^{+}$amplitude in the (spin-singlet) $\otimes$ (valley-triplet) channel, which vanishes at high temperatures, was shown in Ref. 18 to be generated under the RG transformations when $T \lesssim T_{v}$. (To emphasize that $\Gamma_{s-}^{+}$arises as an independent scaling variable only when the valley sub-bands are split, it is designated as $\Gamma_{v} \equiv \Gamma_{s-}^{+}$.) This is a generic feature of mutliband systems with sub-band splittings, it was first discussed in Ref. 17 in the opposite case $T_{v} \lesssim T \lesssim T_{z}$ where the relevant amplitude is $\Gamma_{1+}^{-}$, with the spin and valley indices interchanged.

The splitting of the 15 amplitudes below $T_{v}$ are shown schematically in Fig. 1. The solid horizontal lines mark the relevant amplitudes while the dashed lines mark the irrelevant ones. The degenerate amplitudes under the RG flow are grouped together with the degeneracy indicated in curly brackets. At the lowest temperature $T \lesssim T_{\perp}$, when the two valleys are strongly mixed, only the valleysinglet propagator $\mathcal{D}_{+}$remains gapless. Hence, only the amplitudes in the valley-singlet channel $\Gamma_{+}$survives.

Clearly, the number of relevant $e$-e scattering amplitudes in a multiband system at a given scale is sensitive to the splitting and the interband scattering rates within the bands. Calculations in different temperature regimes in the presence of spin-splitting are detailed below. [Since it is seen experimentally that the phase breaking rate saturates at low temperatures for low electron densities ${ }^{19}$, where the results obtained in this paper are most relevant, the contribution from the cooperon (particleparticle) channel has been suppressed in the calculations.]

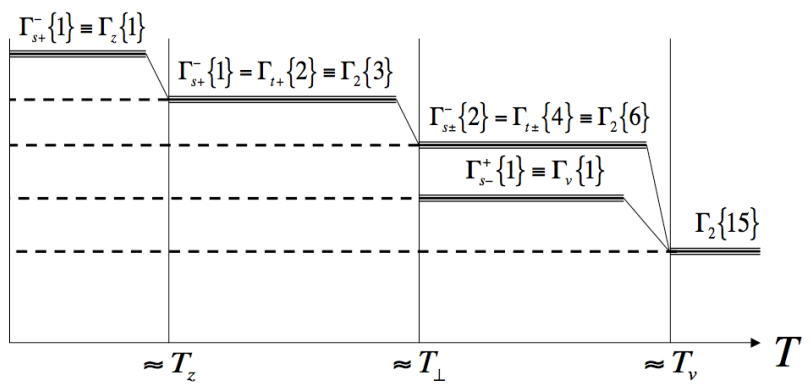

FIG. 2: Schematics showing the classification of the relevant $e$-e scattering amplitudes for weak spin-splitting, $T_{z} \lesssim T_{\perp}, T_{v}$, as function of temperature.

Fig. 2 shows schematically the effect of a weak magnetic field $T_{z} \lesssim T_{\perp}, T_{v}$ on the amplitudes. The spin gap suppresses the singular corrections in the spin-triplet channels, hence only $\Gamma_{s+}^{-}\left(\right.$and $\left.\Gamma_{s+}^{+}\right)$develops singular diffusion corrections. The amplitude is designated as $\Gamma_{z} \equiv \Gamma_{s+}^{-}$to emphasize that $T \lesssim T_{z}$.

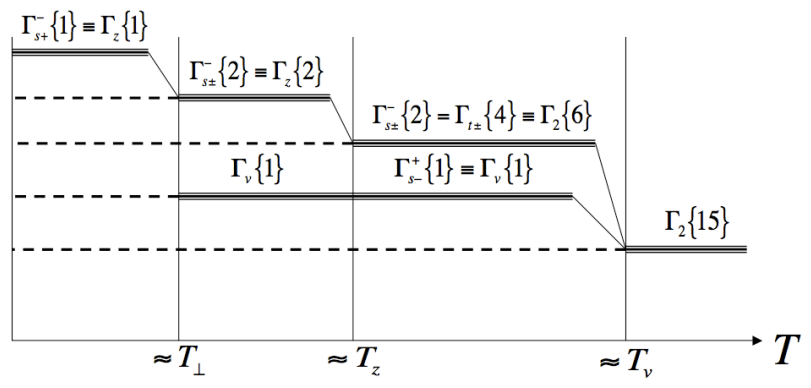

FIG. 3: Schematics showing the classification of the relevant $e$-e scattering amplitudes for intermediate values of the spinsplitting, $T_{\perp} \lesssim T_{z} \lesssim T_{v}$, as function of temperature.

Fig. 3 shows schematically the relevant amplitudes for intermediate values of the spin-splitting $T_{\perp} \lesssim T_{z} \lesssim T_{v}$. As in Fig. 2, the spin-triplet channels, $\Gamma_{t}$, are irrelevant below $T_{z}$ due to the gap in the $\mathcal{D}_{t}$ propagators. As a result, the number of relevant amplitudes (not all degenerate) reduces from seven for $T \gtrsim T_{z}$ to three for $T \lesssim T_{z}$.

\section{SCALING EQUATIONS}

The RG equations in each of the temperature intervals shown in Figs. 2 and 3 are derived below. The relevant equations when spin-splitting can be ignored, $T \gtrsim T_{z}$, have been derived in detail in Ref. 18. The logarithmic corrections are presented here in sections II A and II B after including the spin degrees of freedom explicitly. 


$$
\text { A. } T_{\perp}, T_{z} \lesssim T \lesssim T_{v}
$$

Since the $\mathcal{D}_{\perp}$ modes are gapped for $T \lesssim T_{v}$, their contributions are non-singular and hence dropped. All other modes are effectively gapless when $T \gtrsim T_{z}, T_{\perp}$. The gapless propagators are set equal to $\mathcal{D}_{s \pm}^{\sigma}=\mathcal{D}_{t \pm} \equiv \mathcal{D}(q, \omega)=$ $1 /\left(D q^{2}+z \omega\right)$, where $D$ is the renormalized diffusion constant and $z$ parametrizes the relative scaling of the frequency with respect to the length scale ${ }^{11,20}$. Both $D$ and $z$ acquire diffusion corrections in an interacting system. $\left(z=1\right.$ for a non-interacting system ${ }^{3}$.)

The nature of the gapless diffusion modes induce the following relations on the amplitudes: $\Gamma_{t+}=\Gamma_{t-} \equiv \Gamma_{2}$, $\delta \Gamma_{1+}^{+}=\delta \Gamma_{1-}^{+}$and $\delta \Gamma_{1 \pm}^{-}=0$. Since $\delta \Gamma_{1 \pm}^{-}=0$, the amplitudes $\Gamma_{s \pm}^{-}=\Gamma_{2}$ are degenerate. The diffusion corrections in terms of these variables take the form ${ }^{18}$

$$
\begin{aligned}
\frac{\delta D}{D} & =-\frac{4}{\nu} \iint\left(\Gamma_{1-}^{+}+\Gamma_{1+}^{+}-2 \Gamma_{2}\right) \mathcal{D}^{3}(q, \omega) D q^{2} \\
\delta z & =-\frac{1}{\pi \nu} \int \frac{d^{2} q}{(2 \pi)^{2}}\left(\Gamma_{1-}^{+}+\Gamma_{1+}^{+}-2 \Gamma_{2}\right) \mathcal{D}(q, 0) \\
\delta \Gamma_{2} & =\frac{1}{\pi \nu} \int \frac{d^{2} q}{(2 \pi)^{2}}\left(\Gamma_{1-}^{+}+\Gamma_{1+}^{+}\right) \mathcal{D}(q, 0)+8 \Psi\left(\Gamma_{2}\right),(1 \\
\delta \Gamma_{1 \pm}^{+} & =\frac{1}{2 \pi \nu} \int \frac{d^{2} q}{(2 \pi)^{2}} \Gamma_{2} \mathcal{D}(q, 0)+2 \Psi\left(\Gamma_{2}\right)
\end{aligned}
$$

The single integral is defined as $\int=d^{2} q /(2 \pi)^{2}$ and the double integral as $\iint=\int d^{2} q /(2 \pi)^{2} \int d \omega /(2 \pi)$. The density of states per spin and valley $\nu=m / 2 \pi$. The contributions of the "ring" diagrams ${ }^{11}$ equals $\Psi\left(\Gamma_{2}\right)$, where (see Fig. 5 in 18)

$$
\begin{aligned}
\Psi\left(\Gamma_{2}\right)= & +\frac{1}{\nu} \iint \Gamma_{2}\left[\Gamma_{2} \mathcal{D}^{2}\right]-\frac{1}{2}\left[\Gamma_{2}^{2} \mathcal{D}^{2}\right] \\
& -\frac{1}{\nu} \iint \omega \Gamma_{2}\left[\Gamma_{2}^{2} \mathcal{D}^{3}\right]-\omega \Gamma_{2}^{2}\left[\Gamma_{2} \mathcal{D}^{3}\right] \\
& -\frac{1}{2 \nu} \iint \omega^{2} \Gamma_{2}^{2}\left[\Gamma_{2}^{2} \mathcal{D}^{4}\right]
\end{aligned}
$$

As noted already, the relevance of the $\Gamma_{1-}^{+}$amplitude in the temperature range $T_{\perp} \lesssim T \lesssim T_{v}$ is specific to problems with split-bands in a multivalley system. Although the corrections $\delta \Gamma_{1+}^{+}=\delta \Gamma_{1-}^{+}$for $T \lesssim T_{v}$, their initial values are different. The amplitude $\Gamma_{1-}^{+}=0$ when $T \gtrsim T_{v}$, while the singlet amplitude $\Gamma_{1+}^{+}$is special as it combines with the static limit of the Coulomb interaction, denoted here as $\Gamma_{0+}^{+}$(it is conventionally denoted simply as $\Gamma_{0}$ in a single valley system with degenerate spin bands ${ }^{3}$ ). The $\Gamma_{1+}^{+}$amplitude appearing in (1a)-(1c) are to be replaced by its long-ranged value

$$
\Gamma_{1+}^{+} \longrightarrow \Gamma_{1}^{L R}=\Gamma_{0+}^{+}+\Gamma_{1+}^{+} .
$$

When combined with the $\Gamma_{2}$ amplitude, the long-ranged singlet amplitude is given as: $\Gamma_{s}^{L R}=\Gamma_{2}-4 \Gamma_{1}^{L R}$. (To be consistent with the notations in this paper, $\Gamma_{s}^{L R}$ is defined with an extra factor of -4.) It is easily verified by combining Eqs. (1b)-(1d) that the singlet combination $\delta\left(z+\Gamma_{s}^{L R}\right)=0$ is satisfied at all length scales, provided the corrections to the static amplitude $\delta \Gamma_{0+}^{+}=0$. This is a well established result, with great importance for the general structure of the theory 11,12 .

Having obtained the leading logarithmic corrections, the scaling equations are derived to first order in the dimensionless resistance $\rho=1 / 4\left(2 \pi^{2} \nu D\right)$ and to all orders in the $e-e$ interaction amplitudes by performing the ladder summations described in Fig. 6 in Ref. 18. It amounts to replacing the static amplitudes $\Gamma_{i}$ by the dynamical amplitudes $U_{i}(q, \omega)$ :

$$
U_{i}(q, \omega)=\Gamma_{i} \frac{\mathcal{D}_{i}(q, \omega)}{\mathcal{D}(q, \omega)},
$$

where, the propagators $\mathcal{D}_{i}$ are defined as

$$
\mathcal{D}_{i}(q, \omega)=\frac{1}{D q^{2}+\left(z+\Gamma_{i}\right) \omega} .
$$

The amplitudes $\Gamma_{i}$ represents $\Gamma_{2}, \Gamma_{s-}^{+}$and $\Gamma_{s}^{L R}$. Note that since the leading logarithmic corrections involve only one momentum integration, it generates only one factor of $1 / D$. The corrections are therefore limited to the first order in resistance $\rho$ (disorder). The limitation on the number of momentum integrations constraints the number of $e$-e vertices in the skeleton diagrams. The ladder sums extend the skeleton diagrams to all orders in $\Gamma_{i}$ without changing the number of momentum integrations. Note, however, that only those interaction vertices involving frequency integrations can be extended to include dynamical effects. These amplitudes are enclosed in square brackets in Eq. (2). Substituting the $\Gamma_{2}$ amplitudes in the square brackets with $U_{2}$ and performing the $q, \omega$ integrals leads to the very simple expression ${ }^{8,11}$ :

$$
\Psi\left(\Gamma_{2}\right)=\left(\frac{\Gamma_{2}^{2}}{z}\right) \times \frac{\rho}{2} \log \left(\frac{1}{T \tau}\right)
$$

The remaining single integrals $\int d^{2} q \mathcal{D}(q, 0)$ involving only momentum integrations are easily evaluated to give

$$
\frac{1}{\pi \nu} \int \frac{d^{2} q}{(2 \pi)^{2}} \mathcal{D}(q, 0)=2 \rho \log \left(\frac{1}{T \tau}\right) .
$$

The integrals in $\delta D$ containing $\omega$ integrations remain to be evaluated. Before the integrals can be done, the $\Gamma_{1+}^{+}$ amplitude is replaced with $\Gamma_{1}^{L R}$ following Eq. (3), after which the amplitudes $\Gamma_{1}^{L R}, \Gamma_{1-}^{+}$and $\Gamma_{2}$ are rearranged to form $\Gamma_{s}^{L R}$ and $\Gamma_{s-}^{+}$and $\Gamma_{2}$ and then extended to $U_{s}^{L R}, U_{s-}^{+}$ and $U_{2}$, respectively.

When the equation for $\rho$ is expressed in terms of the scaling variables, $\gamma_{2}=\Gamma_{2} / z$ and $\gamma_{v}=\Gamma_{s-}^{+} / z$, the equations for $\rho, \gamma_{2}$ and $\gamma_{v}$ form a closed set of equations independent of $z$. The final RG equations in the range 
$T_{\perp}, T_{z} \lesssim T \lesssim T_{v}$ are given below with the scale $\xi$ defined to $\operatorname{logarithmic}$ accuracy as $\xi=\log (1 / T \tau)$.

$$
\begin{aligned}
\frac{d \rho}{d \xi} & =\rho^{2}\left(1-\Phi\left(\gamma_{v}\right)-6 \Phi\left(\gamma_{2}\right)\right) \\
\frac{d \gamma_{2}}{d \xi} & =\frac{\rho}{2}\left[\left(1+\gamma_{2}\right)^{2}+\left(1+\gamma_{2}\right)\left(\gamma_{2}-\gamma_{v}\right)\right] \\
\frac{d \gamma_{v}}{d \xi} & =\frac{\rho}{2}\left(1+\gamma_{v}\right)\left(1-\gamma_{v}-6 \gamma_{2}\right) \\
\frac{d \ln z}{d \xi} & =-\frac{\rho}{2}\left(1-\gamma_{v}-6 \gamma_{2}\right)
\end{aligned}
$$

The function $\Phi(\gamma)$ is defined as

$$
\Phi(\gamma)=\left(1+\frac{1}{\gamma}\right) \log (1+\gamma)-1
$$

As described in Fig. 3, the 15 degenerate amplitudes for $T \gtrsim T_{v}$ split into six $\Gamma_{2}$ and one $\Gamma_{v}$ amplitude when $T \lesssim T_{v}$. [This splitting of the amplitudes is generic to multiband systems with subband splittings. The same equations are obtained when instead of the valley bands, the spin bands are split ${ }^{17}$, i.e., $T_{v} \lesssim T \lesssim T_{z}$.] Note that $\gamma_{v}$ coincides with $\gamma_{2}$ when $T \approx T_{v}$.

\section{B. $T_{z} \lesssim T \lesssim T_{\perp}, T_{v}$}

The relevant amplitudes in the presence of strong valley mixing $\left(T \lesssim T_{\perp}\right)$ correspond to scattering in the valley-singlet channels, $\Gamma_{s+}^{-}$and $\Gamma_{t+}$. Since $\delta \Gamma_{1+}^{-}=0$ vanishes in the absence of spin-splitting $\left(T \gtrsim T_{z}\right)$, it follows that the amplitudes $\Gamma_{s+}^{-}=\Gamma_{t+}=\Gamma_{2}$ are all equal and satisfy the equations ${ }^{18}$

$$
\begin{aligned}
\frac{\delta D}{D} & =-\frac{4}{\nu} \iint\left(\Gamma_{1+}^{+}-\Gamma_{2}\right) \mathcal{D}^{3}(q, \omega) D q^{2}, \\
\delta z & =-\frac{1}{\pi \nu} \int \frac{d^{2} q}{(2 \pi)^{2}}\left(\Gamma_{1+}^{+}-\Gamma_{2}\right) \mathcal{D}(q, 0), \\
\delta \Gamma_{2} & =\frac{1}{\pi \nu} \int \frac{d^{2} q}{(2 \pi)^{2}} \Gamma_{1+}^{+} \mathcal{D}(q, 0)+4 \Psi\left(\Gamma_{2}\right), \\
\delta \Gamma_{1+}^{+} & =\frac{1}{4 \pi \nu} \int \frac{d^{2} q}{(2 \pi)^{2}} \Gamma_{2} \mathcal{D}(q, 0)+\Psi\left(\Gamma_{2}\right) .
\end{aligned}
$$

The coefficient of $\Gamma_{2}$ and the ring diagrams $\Psi\left(\Gamma_{2}\right)$ in (10a)-(10c) are suppressed by a factor two when compared with (1a)-(1c) since they no longer contain a valley sum. The corrections to $\Gamma_{1 \alpha}^{\sigma}$ in (10d) already do not carry a valley sum, only half the amplitude involving the same valley, however, acquires corrections when the valley-bands are mixed, which accounts for the overall factor of half when compared with (1d). Note that the condition $\delta\left(z+\Gamma_{s}^{L R}\right)=0$ is satisfied. Following the procedure described in section II A, the RG equations read

$$
\begin{aligned}
\frac{d \rho}{d \xi} & =\rho^{2}\left(1-3 \Phi\left(\gamma_{2}\right)\right) \\
\frac{d \gamma_{2}}{d \xi} & =\frac{\rho}{2}\left(1+\gamma_{2}\right)^{2} \\
\frac{d \ln z}{d \xi} & =-\frac{\rho}{2}\left(1-3 \gamma_{2}\right)
\end{aligned}
$$

The function $\Phi(\gamma)$ is defined in Eq. (9). As described in Fig. 2, only three of the 15 degenerate amplitudes survive when $T \lesssim T_{\perp}$ when spin-splitting can be neglected $T \gtrsim$ $T_{z}$. The high field cases are discussed below, i.e., $T \lesssim T_{z}$.

$$
\text { C. } T_{\perp} \lesssim T \lesssim T_{z} \lesssim T_{v}
$$

It should be noted that the results for $T \lesssim T_{z} \lesssim T_{v}$ is equivalent to the situation if the gap scales were reversed, i.e., $T \lesssim T_{v} \lesssim T_{z}$, provided of course the spin and valley indices are interchangeable, which is the case when $T \gtrsim$ $T_{\perp}$. The RG equations for $T_{v} \lesssim T_{z}$ are derived in Ref. 17 . The opposite situation $T_{z} \lesssim T_{v}$ is derived here.

When $T \lesssim T_{z}$, the $\mathcal{D}_{t \pm}$ propagators are gapped, and hence the corrections in the $S_{z}= \pm 1$ channel are nonsingular. The corresponding amplitudes $\Gamma_{t \pm}$ are therefore irrelevant at these temperatures, which reduces the number of relevant interaction amplitudes by four. Furthermore, the amplitude $\Gamma_{1+}^{-}$acquires diffusion corrections ${ }^{17}$ when $T \lesssim T_{z}$ in the same way that $\Gamma_{1-}^{+}$does when $T \lesssim T_{v}$. Since $T \lesssim T_{z}$ and $T_{v}$, the amplitude $\Gamma_{1-}^{-}$ also acquires logarithmic corrections. As a result, both $\Gamma_{s \pm}^{-}$are different from $\Gamma_{2}$ when $T \lesssim T_{z}, T_{v}$. After including the contributions from $\Gamma_{1 \alpha}^{\sigma}$, the diffusion corrections for $T \lesssim T_{z}$ take the form:

$$
\begin{aligned}
\frac{\delta D}{D} & =-\frac{4}{\nu} \iint\left(\sum_{\alpha, \sigma= \pm} \Gamma_{1 \alpha}^{\sigma}-\Gamma_{2}\right) \mathcal{D}^{3}(q, \omega) D q^{2} \\
\delta z & =-\frac{1}{\pi \nu} \int\left(\sum_{\alpha, \sigma= \pm} \Gamma_{1 \alpha}^{\sigma}-\Gamma_{2}\right) \mathcal{D}(q, 0) \\
\delta \Gamma_{2} & =\frac{1}{\pi \nu} \int \sum_{\alpha, \sigma= \pm} \Gamma_{1 \alpha}^{\sigma} \mathcal{D}(q, 0)+4 \Psi\left(\Gamma_{2}\right) \\
\delta \Gamma_{1 \alpha}^{\sigma} & =\frac{1}{4 \pi \nu} \int \Gamma_{2} \mathcal{D}(q, 0)+\Psi\left(\Gamma_{2}\right) .
\end{aligned}
$$

The coefficient of $\Gamma_{2}$ and the ring diagrams $\Psi\left(\Gamma_{2}\right)$ in (12a)-(12c) are suppressed by a factor two when compared with (1a)-(1c) since they no longer contain a spin sum. The corrections to $\Gamma_{1 \alpha}^{\sigma}$ in (12d) already do not carry a spin sum. Only half the amplitude involving the same spin, however, acquires corrections when the spinbands are split, which accounts for the overall factor of half when compared with (1d). Since $\delta \Gamma_{1+}^{-}=\delta \Gamma_{1-}^{-}$, the amplitudes, after combining with $\Gamma_{2}$, can be grouped together as $\Gamma_{s \alpha}^{-} \equiv \Gamma_{z}$. Extending the singlet amplitude $\Gamma_{1+}^{+}$ 
to include the static long ranged part of the Coulomb interactions $\Gamma_{1}^{L R}$ as discussed in Eq. (3) and using the identity $\Gamma_{2}-\sum_{\alpha, \sigma} \Gamma_{1 \alpha}^{\sigma}=\sum_{\alpha, \sigma} \Gamma_{s \alpha}^{\sigma} / 4$, the amplitude $\Gamma_{2}$ can be eliminated from Eqs. (12a)-(12d) in favor of the amplitudes $\Gamma_{s}^{L R}, \Gamma_{z}$ and $\Gamma_{v}$ as

$$
\begin{aligned}
\frac{\delta D}{D} & =\frac{1}{\nu} \iint\left(\Gamma_{s}^{L R}+\Gamma_{v}+2 \Gamma_{z}\right) \mathcal{D}^{3}(q, \omega) D q^{2} \\
\delta z & =\frac{1}{4 \pi \nu} \int\left(\Gamma_{s}^{L R}+\Gamma_{v}+2 \Gamma_{z}\right) \mathcal{D}(q, 0) \\
\delta \Gamma_{z} & =\delta \Gamma_{v}=\delta \Gamma_{s}^{L R}=-\delta z
\end{aligned}
$$

Combining (12c) and (12d) to give Eq. (13c) is possible only because the $\Psi\left(\Gamma_{2}\right)$ contribution cancels exactly when the sum over opposite spin-projections are suppressed due to spin-splitting ${ }^{7}$. Also note in $(13 \mathrm{c})$, that the singlet combination $\delta\left(z+\Gamma_{s}^{L R}\right)=0$ holds explicitly, as needed for the consistency of the RG theory ${ }^{8,12}$.

The RG equations are obtained by evaluating the integrals after extending the static amplitudes by the dynamical amplitudes $U_{i}$ defined in Eq. (4). The RG equations for $T_{\perp} \lesssim T \lesssim T_{z} \lesssim T_{v}$ are

$$
\begin{aligned}
\frac{d \rho}{d \xi} & =\rho^{2}\left(1-\Phi\left(\gamma_{v}\right)-2 \Phi\left(\gamma_{z}\right)\right) \\
\frac{d \gamma_{z}}{d \xi} & =\frac{\rho}{2}\left(1+\gamma_{z}\right)\left(1-\gamma_{v}-2 \gamma_{z}\right) \\
\frac{d \gamma_{v}}{d \xi} & =\frac{\rho}{2}\left(1+\gamma_{v}\right)\left(1-\gamma_{v}-2 \gamma_{z}\right) \\
\frac{d \ln z}{d \xi} & =-\frac{\rho}{2}\left(1-\gamma_{v}-2 \gamma_{z}\right)
\end{aligned}
$$

As described in Fig. 3, the four $\Gamma_{t \pm}$ amplitudes are suppressed when $T \lesssim T_{z}$, leaving two $\Gamma_{z}$ amplitudes, which evolve away from $\Gamma_{2}$. Note that $\gamma_{z} \approx \gamma_{2}$ when $T \approx$ $T_{z}$, while $\gamma_{v} \approx \gamma_{2}$ when $T \approx T_{v}$. [The RG equations when spin-splitting is large, $T \lesssim T_{v} \lesssim T_{z}$, take the same form as Eqs. (14a)-(14d) provided the spin and valley indices are interchanged; see Ref. 17 for details.]

$$
\text { D. } T \lesssim T_{\perp}, T_{z}, T_{v}
$$

The two valleys are strongly mixed when $T \lesssim T_{\perp}$, leaving only the valley-singlet propagators $\mathcal{D}_{s+}^{\sigma}$ gapless. Hence, only $\Gamma_{s+}^{-}=\Gamma_{z}$ and $\Gamma_{1+}^{+}$, survive at low temperatures. The corresponding diffusion corrections read

$$
\begin{aligned}
\frac{\delta D}{D} & =-\frac{4}{\nu} \iint\left(\Gamma_{1+}^{-}+\Gamma_{1+}^{+}-\frac{1}{2} \Gamma_{2}\right) \mathcal{D}^{3}(q, \omega) D q^{2} \\
\delta z & =-\frac{1}{\pi \nu} \int\left(\Gamma_{1+}^{-}+\Gamma_{1+}^{+}-\frac{1}{2} \Gamma_{2}\right) \mathcal{D}(q, 0) \\
\delta \Gamma_{2} & =\frac{1}{\pi \nu} \int\left(\Gamma_{1+}^{-}+\Gamma_{1+}^{+}\right) \mathcal{D}(q, 0)+2 \Psi\left(\Gamma_{2}\right) \\
\delta \Gamma_{1+}^{\sigma} & =\frac{1}{8 \pi \nu} \int \Gamma_{2} \mathcal{D}(q, 0)+\frac{1}{2} \Psi\left(\Gamma_{2}\right)
\end{aligned}
$$

All terms involving $\Gamma_{2}$ amplitudes are suppressed by a factor of two in (15a)-(15c) compared to (12a)-(12c) due to the suppression of the $\Gamma_{t-}$ amplitudes, which are irrelevant when $T \lesssim T_{\perp}$. The equations can be simplified in terms of the amplitudes $\Gamma_{s}^{L R}$ and $\Gamma_{z}$ as

$$
\begin{aligned}
\frac{\delta D}{D} & =\frac{1}{\nu} \iint\left(\Gamma_{s}^{L R}+\Gamma_{z}\right) \mathcal{D}^{3}(q, \omega) D q^{2} \\
\delta z & =\frac{1}{4 \pi \nu} \int\left(\Gamma_{s}^{L R}+\Gamma_{z}\right) \mathcal{D}(q, 0) \\
\delta \Gamma_{z} & =\delta \Gamma_{s}^{L R}=-\delta z
\end{aligned}
$$

Note again that the condition $\delta\left(z+\Gamma_{s}^{L R}\right)=0$ is satisfied. Following the procedure followed in the previous sections, the RG equations for $T \lesssim T_{\perp}, T_{z}, T_{v}$ are

$$
\begin{aligned}
\frac{d \rho}{d \xi} & =\rho^{2}\left(1-\Phi\left(\gamma_{z}\right)\right) \\
\frac{d \gamma_{z}}{d \xi} & =\frac{\rho}{2}\left(1+\gamma_{z}\right)\left(1-\gamma_{z}\right) \\
\frac{d \ln z}{d \xi} & =-\frac{\rho}{2}\left(1-\gamma_{z}\right)
\end{aligned}
$$

These equations coincide with the results obtained in the case of a single valley with spin-splitting studied in Ref. 20. Strong intervalley scattering for $T \lesssim T_{\perp}$ mixes the two valleys to effectively produce a single valley.

\section{CONCLUSIONS}

The derivation of the scaling equations in Sec. II were carried out keeping only the gapless valley and spin channels in each temperature interval. The scale dependence of the dimensionless resistance $\rho=\left(e^{2} / \pi h\right) R_{\square}$, where $R_{\square}$ is the sheet resistance, is then obtained by integrating the self consistent set of scaling equations separately in each temperature interval and matching the values of the amplitudes and resistance at the boundaries of each interval. Since the intervals are sensitive to the value of $T_{z}$, one obtains in this way $\rho\left(B_{\|}, T\right)$ whose inverse gives $\sigma\left(B_{\|}, T\right)=1 / \rho\left(B_{\|}, T\right)$. This method is, however, not accurate as the crossover regions have finite contributions from the gapped channels and are hence non-universal.

The case of weak spin-splitting $T_{z} \lesssim T$ can be treated fairly accurately, however. In this case the sensitivity to $B_{\|}$arises only from the presence of a weak spin-gap in the triplet channels below the scale set by $T$. Hence, subtracting $\sigma(0, T)$ from $\sigma\left(B_{\|}, T\right)$ captures only the contributions originating from the suppression of the triplet channels. The explicit form $\Delta \sigma\left(B_{\|}, T\right)$ for the single valley case was derived in Refs. 5,14 in the limit $T_{z} \ll T$. It is straightforward to extend the results to include the valley degrees of freedom by keeping track of the number of $\Gamma_{t}$ amplitudes in a given temperature interval as ${ }^{1}$

$$
\Delta \sigma\left(B_{\|}, T\right)=-0.091 \frac{e^{2}}{2 \pi h} N_{t} \gamma_{2}\left(\gamma_{2}+1\right)\left(T_{z} / T\right)^{2}
$$


The number of relevant $\Gamma_{t}$ amplitudes, $N_{t}$ can be read off directly from Figs. 1, 2 and 3 as $N_{t}=2$ for $T_{z} \lesssim T \lesssim T_{\perp}$, $N_{t}=4$ for $T_{z} \lesssim T \lesssim T_{v}$ and $N_{t}=8$ for $T \gtrsim T_{v}, T_{z}$.

To summarize, RG equations in the presence of spinsplitting induced by a parallel magnetic field have been obtained in a two valley system in the valley-split and strong intervalley scattering regimes. The behavior of the magnetoconductance in the limit of weak magnetic field are discussed.

\section{ACKNOWLEDGEMENTS}

The author would like to thank A. M. Finkelstein and S. V. Kravchenko for fruitful discussions. This work was supported by DOE Grant No. DOE-FG02-84-ER45153.
* Electronic address: punnoose@sci.ccny.cuny.edu

1 S. Anissimova, S. V. Kravchenko, A. Punnoose, A. M. Finkel'stein, and T. M. Klapwijk, Nature Physics 3, 707 (2007).

2 D. A. Knyazev, O. E. Omel'yanovskii, V. M. Pudalov, and I. S. Burmistrov, JETP Lett. 84, 662 (2007).

3 B. L. Altshuler and A. G. Aronov, Modern Problems in Condensed Matter Physics (Elsevier, North Holland, 1985), chap. Electron-Electron Interactions in Disordered Systems, p. 1.

4 A. Kawabata, J. Phys. Soc. Jpn. 50, 2461 (1981).

${ }^{5}$ P. A. Lee and T. V. Ramakrishnan, Phys. Rev. B 26, 4009 (1982).

6 A. M. Finkel'stein, JETP Lett. 37, 517 (1983).

7 A. M. Finkel'stein, Zh. Eksp. Teor. Fiz. 86, 367 (1984).

8 C. Castellani, C. D. Castro, P. A. Lee, and M. Ma, Phys. Rev. B 30, 527 (1984).

${ }^{9}$ H. Fukuyama, J. Phys. Soc. Jpn. 49, 649 (1980).

${ }^{10}$ H. Fukuyama, J. Phys. Soc. Jpn. 50, 3562 (1981).
11 A. M. Finkel'stein, Sov. Phys. JETP 57, 97 (1983).

12 A. M. Finkel'stein, Sov. Sci. Rev. A, Phys. Rev. 14, 1 (1990).

13 R. Raimondi, C. Castellani, and C. D. Castro, Phys. Rev. B 42, 4724 (1990).

14 C. Castellani, C. D. Castro, and P. A. Lee, Phys. Rev. B 57, R9381 (1998).

15 S. A. Vitkalov, K. James, B. N. Narozhny, M. P. Sarachik, and T. M. Klapwijk, Phys. Rev. B 67, 113310 (2003).

16 A. Y. Kuntsevich, N. N. Klimov, S. A. Tarasenko, N. S. Averkiev, V. M. Pudalov, H. Kojima, and M. E. Gershenson, Phys. Rev. B 75, 195330 (2007).

17 I. S. Burmistrov and N. M. Chtchelkatchev, Phys. Rev. B 77, 195319 (2008).

18 A. Punnoose, Phys. Rev. B 81, 035306 (2010).

19 M. Rahimi, S. Anissimova, M. R. Sakr, S. V. Kravchenko, and T. M. Klapwijk, Phys. Rev. Lett. 91, 116402 (2003).

20 A. M. Finkel'stein, Z. Phys. B 56, 189 (1984). 